\title{
Preparation of 10-hydroxycamptothecin-loaded glycyrrhizic acid-conjugated bovine serum albumin nanoparticles for hepatocellular carcinoma-targeted drug delivery
}

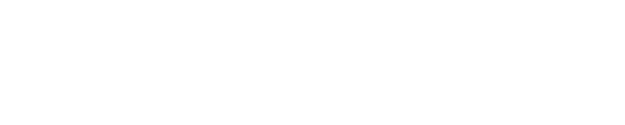

Yuangang $\mathrm{Zu}$

Li Meng

Xiuhua Zhao

Yunlong $\mathrm{Ge}$

Xinyang Yu

Yin Zhang

Yiping Deng

Key Laboratory of Forest Plant Ecology, Northeast Forestry University, Ministry of Education, Harbin, People's Republic of China
Correspondence: Yuangang Zu; Xiuhua Zhao

Key Laboratory of Forest Plant Ecology, Northeast Forestry University,

Ministry of Education, Harbin, Heilongjiang I50040,

People's Republic of China

Tel +86 45I 821915I7

Fax $+8645 I 82102082$

Email zygorl@yahoo.com.cn;

xiuhuazhao@nefu.edu.cn
Introduction: The livertaxis of glycyrrhizic acid-conjugated bovine serum albumin (GL-BSA) has been reported in the literature. Now, in this paper, we describe a novel type of drug-targeted delivery system containing 10-hydroxycamptothecin (HCPT) with liver tumor targeting.

Methods: First, GL was coupled to BSA then HCPT was encapsulated in GL-BSA by highpressure homogenization emulsification. In the experimental design, the influencing variables on particle size and drug loading efficiency were determined to be BSA concentration, volume ratio of water to organic phase, and speed and speed duration of homogenization as well as homogenization pressure and the number of times homogenized at certain pressures. Particle size plays an important role in screening optimal conditions of nanoparticles preparation. Characteristics of 10-hydroxycamptothecin-loaded glycyrrhizic acid-conjugated bovine serum albumin nanoparticles (GL-BSA-HCPT-NPs), such as the drug encapsulation efficiency, drug loading efficiency, and GL-BSA content were studied. In addition, the morphology of the nanoparticles (NPs) and weight loss rate were determined and Fourier transform infrared spectroscopy, X-ray diffraction spectroscopy, and thermal analysis performed.

Results: The average particle size of the sample NPs prepared under optimal conditions was $157.5 \mathrm{~nm}$ and the zeta potential was $-22.51 \pm 0.78 \mathrm{mV}$; the drug encapsulation efficiency and drug loading efficiency were $93.7 \%$ and $10.9 \%$, respectively. The amount of GL coupling to BSA was $98.26 \mu \mathrm{g} / \mathrm{mg}$. Through physical property study of the samples, we determined that the HCPT had been successfully wrapped in GL-BSA. In vitro drug-release study showed that the nanoparticles could release the drug slowly and continuously. Hemolysis testing showed the safety of GL-BSA as a novel drug delivery system. The targeting properties of GL-BSA-HCPT-NPs were studied in an in vitro cell uptake study and cell proliferation assay. Cells incubated with GL-BSA-HCPT-NPs and labeled with fluorescein isothiocyanate showed more extensive fluorescence spots and stronger fluorescence intensity than samples without GL conjugation. MTT (3-(4,5-Dimethylthiazol-2-yl)-2,5-diphenyltetrazolium bromide) assay was used to determine the inhibitory rate of the samples. It was found that the inhibitory rate of GL-BSA-HCPT-NPs develops as concentration rises. Further, the inhibitory rate of GL-BSA-HCPT-NPs was higher at the same concentration and had a lower half maximal inhibitory concentration value than the other samples. The half maximal inhibitory concentration values of GL-BSA-HCPT-NPs, BSA-HCPT-NPs, and HCPT sodium were $0.78 \pm 0.015,1.62 \pm 0.039$, and $7.93 \pm 0.255 \mu \mathrm{g} /$ $\mathrm{mL}$, respectively.

Conclusion: The results of this study show GL-BSA-HCPT to be a promising new vehicle for hepatocellular carcinoma-targeting therapy.

Keywords: HCPT, BSA, GL, high-pressure homogenization emulsification 


\section{Introduction}

Camptothecin (CPT), an alkaloid isolated from the Chinese plant Camptotheca acuminata, is a potent agent against a wide spectrum of human cancers. ${ }^{1} \mathrm{CPT}$ and related analogs may lead to the death of tumor cells by targeting the nuclear enzyme, topoisomerase I, and inhibiting the regulation of the cleaved DNA strand. ${ }^{2}$ One camptothecin analog, 10-hydroxycamptothecin (HCPT; Figure 1A), has excellent antitumor activity against hepatoma, gastric carcinoma, leukemia, and head and neck tumors. ${ }^{3,4}$ Further, it is more potent and less toxic than CPT. ${ }^{5,6}$

It is well known that the poor aqueous solubility of drug candidates is a major challenge for pharmaceutical scientists involved in drug development. ${ }^{7}$ As HCPT has poor water solubility and there are other physiologically more acceptable solvents, as yet, there have been limited clinical applications of HCPT. The lactone ring of HCPT is open in an alkaline environment and the sodium salt of HCPT (ring-opened form) is very soluble in water. However, HCPT sodium is much less active than HCPT. ${ }^{8}$ It also causes adverse effects such as myelosuppression, hemorrhagic cystitis, vomiting, nausea, and dermatitis. ${ }^{9}$ Many different methods have been used to overcome these limitations, including the formulation of HCPT-containing nanocrystallites, ${ }^{10}$ niosomes, ${ }^{11}$ micelles, ${ }^{4,12}$ and nanoparticles (NPs), which have been somewhat successful. ${ }^{13}$

Many different materials are used as drug carriers, such as chitosan, ${ }^{14}$ poly (lactide-co-glycolide),${ }^{15}$ polybutylcyanoacrylate, ${ }^{16}$ and albumin. Albumin has been used widely in recent years because of its biodegradability, stability, and lower toxicity, ${ }^{17}$ as well as its non-immunogenic properties and biocompatibility. ${ }^{18}$ Similarly, bovine serum albumin (BSA) is widely used for drug delivery because of its abundance, low cost, ease of purification, unusual ligandbinding properties, and its wide acceptance in the pharmaceutical industry. ${ }^{19,20}$ In a study by Yang et al examining a method of wrapping HCPT in BSA, the poor solubility of HCPT was overcome by dissolving it in sodium hydroxide solution. ${ }^{21}$ However, the resulting HCPT sodium had many shortcomings, as already described. Moreover, the drug

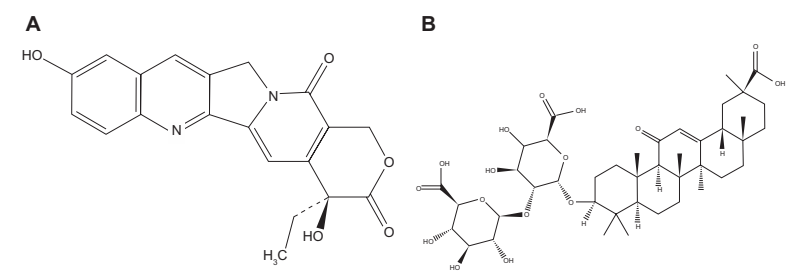

Figure I The structure of (A) 10-hydroxycamptothecin and (B) glycyrrhizic acid. entrapment efficiency and drug loading efficiency were low, and the BSA significantly retarded the lactone ring opening, ${ }^{22}$ while the drug carrier lacked targeting ability.

Hepatocellular carcinoma is one of the most prevalent causes of cancer-related deaths in the world, particularly in the eastern and southeastern part of Asia and sub-Saharan Africa. ${ }^{23,24}$ Various treatments for liver cancer have been extensively studied, including surgical resection, ${ }^{25}$ radiation therapy, ${ }^{26}$ and drug therapy, ${ }^{27}$ but these treatments have largely proved insufficient. Moreover, it is difficult for the commonly used drugs to accurately reach the lesion due to the detoxification of the liver. Glycyrrhizic acid (GL; Figure 1B) is hepatoprotective ${ }^{28}$ and has been used to clinically treat patients with chronic active hepatitis. ${ }^{29}$ Studies have shown that GL is mainly taken up into the liver ${ }^{30}$ and modified liposomes have displayed good livertaxis. ${ }^{31,32}$ The surface of GL has been modified based on N-caproyl chitosan and confirmed as a vehicle for hepatocyte targeting. ${ }^{33}$ In addition, favorable livertaxis of glycyrrhizic acid-conjugated bovine serum albumin (GL-BSA) has been confirmed in experiments. ${ }^{34}$ Some liver-targeting HCPT delivery systems, such as galactosylated chitosan ${ }^{10}$ and HCPT polybutylcyanoacrylate NPs associated with polybutylpyrolidone ${ }^{16}$ have been reported. However, glycyrrhizic acid-conjugated bovine serum albumin nanoparticles (GL-BSA-NPs) are superior to other delivery systems due to the properties of BSA and their liver-targeting ability.

Taking into account the effects of liver cancer on human health and its difficult treatment, in the study reported here, we attempted to establish a liver cancer-targeted drug delivery system using HCPT to produce 10-hydroxycamptothecinloaded glycyrrhizic acid-conjugated bovine serum albumin nanoparticles (GL-BSA-HCPT-NPs). Thus, in this paper, we describe a novel type of drug-targeted delivery system containing HCPT targeted to liver tumors. First, GL was coupled to BSA then HCPT was encapsulated in the resulting GL-BSA by high-pressure homogenization emulsification. The variables affecting particle size and drug loading efficiency investigated included BSA concentration, volume ratio of water to organic phase, and speed and speed duration of homogenization as well as homogenization pressure and the number of times homogenized at certain pressures. The related features of GL-BSA-HCPT-NPs were fully characterized by scanning electron microscopy (SEM), Fourier transform infrared spectroscopy (FTIR), thermal gravimetry (TG), X-ray diffraction (XRD), and differential scanning calorimetry (DSC); the drug release in vitro was also assessed and hemolysis testing undertaken. Finally, the 
targeting properties of GL-BSA-HCPT-NPs were studied in an in vitro cell uptake study and a cell proliferation assay undertaken.

\section{Materials and methods Materials}

HCPT ( $\geq 98 \%$ ) was provided by Hisun Pharmaceutical (Taizhou, People's Republic of China). BSA was obtained from Sigma-Aldrich (St Louis, MO, USA). Fluorescein isothiocyanate (FITC), Roswell Park Memorial Institute (RPMI) 1640 medium, and trypsin-ethylenediaminetetraacetic acid were purchased from Invitrogen (Carlsbad, CA, USA). MTT (3-(4,5-dimethylthiazol-2-yl)-2,5-diphenyl-2 H-tetrazolium bromide; $98 \%$ purity) was purchased from Sigma-Aldrich. Methanol and acetonitrile were of high-performance liquid chromatography (HPLC) grade. Phosphate-buffered saline (PBS; 0.15 M, pH = 7.2-7.6), trichloromethane, ethanol, and the other reagents were all of analytical grade.

\section{Animals}

New Zealand white rabbits were used for the experiments. Procedures involving animals and their care were conducted in accordance with National Institutes of Health guidelines ${ }^{35}$ and all experiments were carried out in compliance with the guidelines issued by the Ethical Committee of Northeast Forestry University.

\section{Preparation of GL-BSA-HCPT-NPs Preparation of GL-BSA}

The preparation process is referred to in the literature. ${ }^{36}$ Briefly, $4.0 \mathrm{~mL}$ of $\mathrm{MeOH}$ solution containing $59.6 \mathrm{mg}$ GL was added dropwise to a $4.0 \mathrm{~mL}$ water solution of $\mathrm{NaIO}_{4}$ and stirred for 60 minutes. Following this, a carbonate buffer solution $(\mathrm{pH}=9.6,14 \mathrm{~mL})$ containing BSA $(50 \mathrm{mg})$ was added to the mixture. The reaction mixture was adjusted to $\mathrm{pH}=10.0$ with $1 \mathrm{M} \mathrm{Na}_{2} \mathrm{CO}_{3}$ solution and stirred at room temperature for 6 hours then dialyzed for 3 days against water, resulting in the GL-BSA solution. GL-BSA ( $2 \mathrm{~mL}$ ) solution was placed in an $8 \mathrm{~mL}$ vial for freeze-drying. Pre-freezing was carried out at $-40^{\circ} \mathrm{C}$ for 2 hours, then the sample was placed in a vacuum lyophilizer and freeze-dried at $-60^{\circ} \mathrm{C}$ for 48 hours to obtain GL-BSA lyophilized powder.

\section{Preparation of GL-BSA-HCPT NPs}

HCPT was dissolved in a mixture consisting of chloroform and ethanol then dispersed for 5-10 minutes in an Elmasonic TI-H-5 ultrasonication bath (Elma, Singen, Germany). The resultant solution was slowly added dropwise to deionized water containing GL-BSA, mixed with a high-speed FSHII Adjustable High-Speed Homogenizer Stirrer (Jiangsu Zhengji Instruments, Jintan, People's Republic of China) and homogenized in an AH-100D ultra-high-pressure nano homogenizer (ATS Engineering, Shanghai, People's Republic of China), generating the nanoemulsion. The organic phase was removed by rotary evaporation using a Rotary Evaporator R201BL (SENCO Technology, Shanghai, People's Republic of China) at an evaporation temperature of $40^{\circ} \mathrm{C}$, resulting in the desired GL-BSA-HCPT-NP suspension.

During the preparation process, the parameters affecting particle size were defined in experiments. As previously outlined, the parameters included BSA concentration; volume ratio of water to organic phase; volume ratio of water to organic phase; and speed and speed duration of homogenization as well as homogenization pressure and the number of times homogenized at certain pressures.

In this study, a single-factor method was used to determine the optimal conditions. The range of BSA concentrations tested was $0.5-4.0 \mathrm{mg} / \mathrm{mL}$. The ratios of water to organic phase volume tested ranged from 5:1 to $33: 1$. The homogenizer speeds examined ranged from 5000 and 10,000 rpm for between 1 and 12 minutes and homogenization pressures from 400 to 1200 bar were, with samples homogenized between three and 15 times. All results are shown in Table 1.

\section{Lyophilization}

After the organic solvents were removed, the GL-HCPT-BSANP suspension was lyophilized using a Gamma 2-20 apparatus (Martin Christ Gefriertrocknungsanlagen, Osterode am Harz, Germany) to ensure the physical stability of the ultimate product. Two milliliters of the GL-HCPT-BSA suspension were added into an $8 \mathrm{~mL}$ glass vial, then pre-frozen at $-40^{\circ} \mathrm{C}$ for 2 hours then subsequently lyophilized at $-60^{\circ} \mathrm{C}$ for 64 hours to obtain the lyophilized powder.

\section{Characterization of GL-HCPT-BSA-NPs}

Determination of amount of GL associated with BSA

A sample (3 mg) of the lyophilized powder was mixed with $\mathrm{KBr}(200 \mathrm{mg})$ and pressed into tablet form. This was then placed in the sample slot of a Magna-IR 560 ESP Fourier Transform Infrared Spectrometer (Nicolet, Madison, WI, USA) for detection of FTIR spectra. Raw KBr was prepared as background.

In acidic conditions, GL was hydrolyzed to glycyrrhetinic

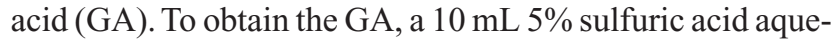


Table I Relationships between parameters and particle size when one variable parameter and others are fixed

\begin{tabular}{llll}
\hline $\begin{array}{l}\text { Order of } \\
\text { evaluation }\end{array}$ & Variable & Level & $\begin{array}{l}\text { Mean particle } \\
\text { diameter } \\
(\mathbf{n m}) \pm \text { SD }\end{array}$ \\
\hline Ist & Concentration & 0.5 & $716.4 \pm 35.9$
\end{tabular}

\begin{tabular}{llll}
\hline Ist & $\begin{array}{l}\text { Concentration } \\
\text { of BSA }(\mathrm{mg} / \mathrm{mL})\end{array}$ & 0.5 & $716.4 \pm 35.9$ \\
& I & $659.2 \pm 33.1$ \\
& 1.5 & $491.6 \pm 23.5$ \\
& 2 & $417.3 \pm 19.8$ \\
& 3 & $348.3 \pm 18.5$ \\
2nd & 4 & $290.0 \pm 14.5$ \\
& & $5: 1$ & $744.3 \pm 33.6$
\end{tabular}

of water and

organic phase $(v / v)$

$3 r d$

Homogenate

speed (rpm)

and time $(\mathrm{min})$

4th

Homogenization pressure (bar) and times
Table I (Continued)

\begin{tabular}{|c|c|c|c|}
\hline $\begin{array}{l}\text { Order of } \\
\text { evaluation }\end{array}$ & Variable & Level & $\begin{array}{l}\text { Mean particle } \\
\text { diameter } \\
(\mathrm{nm}) \pm \mathrm{SD}\end{array}$ \\
\hline \multicolumn{4}{|c|}{600} \\
\hline & & 3 & $\mid 77.7 \pm 8.1$ \\
\hline & & 5 & $190.1 \pm 9.5$ \\
\hline & & 7 & $308.7 \pm 14.5$ \\
\hline & & 9 & $146.3 \pm 7.3$ \\
\hline & & 11 & $173.4 \pm 6.2$ \\
\hline & & 13 & $233.1 \pm 13.5$ \\
\hline & & 15 & $368.7 \pm 14.9$ \\
\hline \multicolumn{4}{|c|}{800} \\
\hline & & 3 & $465.7 \pm 22.8$ \\
\hline & & 5 & $148.9 \pm 7.2$ \\
\hline & & 7 & $144.3 \pm 6.1$ \\
\hline & & 9 & $315.2 \pm 15.3$ \\
\hline & & II & $395.3 \pm 19.5$ \\
\hline & & 13 & $237.7 \pm 11.2$ \\
\hline & & 15 & $355.2 \pm 19.4$ \\
\hline \multicolumn{4}{|c|}{1000} \\
\hline & & 3 & $266.7 \pm 13.3$ \\
\hline & & 5 & $225.9 \pm 11.2$ \\
\hline & & 7 & $4 \mid 2.3 \pm 20.6$ \\
\hline & & 9 & $433.3 \pm 21.6$ \\
\hline & & 11 & $393.7 \pm 19.6$ \\
\hline & & 13 & $343.8 \pm 17.1$ \\
\hline & & 15 & $294.5 \pm 13.5$ \\
\hline \multicolumn{4}{|c|}{1200} \\
\hline & & 3 & $965.8 \pm 48.1$ \\
\hline & & 5 & $221.2 \pm 11.2$ \\
\hline & & 7 & $219.6 \pm 9.3$ \\
\hline & & 9 & $509.7 \pm 24.3$ \\
\hline & & 11 & $430.4 \pm 21.5$ \\
\hline & & 13 & $3 \mid 4.3 \pm 14.2$ \\
\hline & & 15 & $330.9 \pm 14.5$ \\
\hline
\end{tabular}

Abbreviation: SD, standard deviation.

ous solution containing $5 \mathrm{mg}$ GL-BSA lyophilized powder was placed in a $100^{\circ} \mathrm{C}$ water bath for 5 hours. The resulting hydrolysate was placed in a volumetric flask and ethanol was added to reach a final volume of $25 \mathrm{~mL}$. The concentration of GA was determined by a HPLC system equipped with a Jasco UV-975 ultraviolet (UV) detector (Easton, MD, USA) using a Diamonsil $\mathrm{C}_{18}$ column $(4.6 \times 250 \mathrm{~mm}$, $5 \mu \mathrm{m}$; Dikma Technologies, Beijing, People's Republic of China) with a mobile phase consisting of a methanol and $7 \%$ ammonium acetate solution $(86: 14, \mathrm{v} / \mathrm{v})$, flow rate of $1 \mathrm{~mL} /$ $\mathrm{min}$, and UV detection wavelength of $254 \mathrm{~nm}$. The concentration of GA was calculated relative to a GA reference of $y=20877.1355 x+20377.1642$ and $R^{2}=0.9997$ (in which $x$ is the concentration of GA in $\mathrm{mg} / \mathrm{mL}$ and $y$ is the peak area). 


\section{Determination of drug encapsulation \\ efficiency and drug loading efficiency}

The GL-BSA-HCPT-NPs were separated after centrifugation at 10,000 rpm for 20 minutes. Following this, the HCPT concentration of the supernatant was determined by HPLC, performed on the Diamonsil $\mathrm{C}_{18}$ column with a mobile phase of $25 \%$ acetonitrile and $75 \% 0.075 \mathrm{M}$ ammonium acetate buffer $(\mathrm{pH}=6.4)$, flow rate of $1 \mathrm{~mL} / \mathrm{min}$, and UV detective wavelength of $266 \mathrm{~nm} .^{21}$ The concentration in suspension was calculated relative to a HCPT reference of $y=35993992.3465 x+331604.0863 R^{2}=0.9993$ (in which $x$ is the HCPT concentration in $\mathrm{mg} / \mathrm{mL}$ and $y$ is the peak area). Drug loading and encapsulating efficiencies were determined by Equations 1 and 2 .

Drug-loading efficiency (\%)

$$
=\frac{\mathrm{HCPT}_{\text {(total) }}-\mathrm{HCPT}_{\text {(supernatant) }}}{\mathrm{GL}-\mathrm{BSA}-\mathrm{HCPT}-\mathrm{NPs}} \times 100 \%
$$

Drug encapsulating efficiency (\%)

$$
=\frac{\mathrm{HCPT}_{\text {(total) }}-\mathrm{HCPT}_{\text {(supernatant) }}}{\mathrm{HCPT}_{\text {(total) }}} \times 100 \%
$$

\section{Morphology, particle size, and zeta potential of the GL-BSA-HCPT-NPs}

A suitable amount of the lyophilized sample was dissolved in water. A drop of the solution was dropped on a round coverslip then blow-dried with sterile wind using a sterile bench, which also prevented dust from affecting the drop. The coverslip coated with the sample was used to investigate the morphology by SEM (Quanta 200, FEI Co., Eindhoven, The Netherlands).

GL-BSA-HCPT-NPs were dispersed in water to attain a concentration of $5 \mathrm{mg} / \mathrm{mL}$. Then $3 \mathrm{~mL}$ of the solution was placed in a sample cell to detect the particle size by laser light scattering and the zeta potential using a ZetaPlus Zeta Potential Analyzer (Brookhaven Instruments, Holtsville, NY, USA).

\section{In vitro stability test}

The particle size and zeta potential of the prepared GL-BSAHCPT-NP solution with a concentration of $5 \mathrm{mg} / \mathrm{mL}$ was then assessed every hour for 16 hours to observe the stability of the sample at a temperature of $25^{\circ} \mathrm{C}$.

\section{Solid state study}

The solid state of the sample was studied using FTIR spectrometry, XRD, TG, and DSC. The transmittance of the sample was detected using FTIR spectrometry, as described in the "Preparation of GL-BSA" section.

The XRD patterns were used to confirm the crystal phase of the NPs. Six milligrams of the sample was weighed into the sample pool using an X'Pert PRO X-ray diffractometer (Philips, Amsterdam, The Netherlands) with a rotating anode and examined in the range of $5^{\circ}<2 \theta<90^{\circ}$ at $40 \mathrm{kV}$ and $30 \mathrm{~mA}$.

Thermal gravimetric analysis of the sample was performed using a Diamond Thermogravimetric/Differential Thermal Analysis system (Perkin-Elmer, Waltham, MA, USA). Ten milligrams of the sample was put into the sample pool and heated from $34^{\circ} \mathrm{C}$ to $600^{\circ} \mathrm{C}$ at the rate of $10^{\circ} \mathrm{C} / \mathrm{min}$.

DSC analysis of the sample was undertaken using a TA-60WS Thermal Analyzer (Shimadzu, Kyoto, Japan). Six milligrams of the sample was weighed into the sample pool to be scanned from room temperature to $350^{\circ} \mathrm{C}$ at a rate of $10^{\circ} \mathrm{C} / \mathrm{min}$.

The thermal gravimetric and DSC analyses of the sample were performed under nitrogen purge.

\section{In vitro release study}

Lyophilized GL-BSA-HCPT-NP powder $(10.9 \%$ [w/w] drug loading) containing $1.2 \mathrm{mg}$ of HPCT was dissolved in $5 \mathrm{~mL}$ of 0.1 M, pH 7.4 PBS. This was then placed in Slide-A-Lyzer ${ }^{\circledR}$ dialysis cassettes (molecular weight cutoff 10,000; Thermo Fisher Scientific, Waltham, MA, USA). Subsequently, the dialysis cassettes were immersed in a $250 \mathrm{~mL}$ beaker containing $200 \mathrm{~mL}$ PBS. The mixture was stirred at a speed of $100 \mathrm{rpm}$ in an incubator shaker at $37^{\circ} \mathrm{C}$. The release buffer $(4 \mathrm{~mL})$ was taken from the beaker and replaced with $4 \mathrm{~mL}$ fresh release buffer at given time intervals, and the samples were shaken continuously over the course of 72 hours.

In addition, the release of HCPT from HCPT sodium and mixture of HCPT sodium and GL-BSA was studied as a positive control. To do this, mixtures were created containing HCPT sodium and HCPT sodium/GL-BSA - each containing the same concentration of HCPT - instead of GL-BSA-HCPT NPs. Both release studies were repeated three times. The concentration of HCPT in the samples was determined by HPLC using the method already described. The regression equation between peak area $(y)$ and HCPT concentration $(x)$ was fitted as $y=35139.2585 x+5889.2281\left(R^{2}=0.9995\right)$. Cumulative release of drug was calculated by the following formula shown in Equations 3, 4, and 5, ${ }^{37}$ in which $C_{i}$ stands for the HCPT concentration of each sample withdrawn at predetermined time intervals, $C_{i}{ }^{\prime}$ represents the increase of 
the HCPT concentration during each time interval, $V$ stands for the volume of the release buffer, $V_{i}$ is for the volume of each withdrawn sample, $M$ is the total HCPT in the sample, and $Q_{i}$ is the accumulative release percentage at a predetermined point in time.

$$
\begin{gathered}
\mathrm{C}_{1}^{\prime}=\mathrm{C}_{1} \\
C_{i+1}^{\prime}=C_{i+1}-\frac{\left(V-V_{i}\right) C_{i}}{V} \\
Q_{i}=\frac{\sum_{i=1}^{i} C_{i}{ }^{\prime} V}{M} \times 100 \%
\end{gathered}
$$

\section{Hemolysis test}

Rabbit blood was used to test the hemolysis effect of GLBSA-HCPT-NPs. Heparinized rabbit whole blood (10 mL) was added to $5 \mathrm{~mL}$ of physiological saline $(5 \mathrm{~mL})$, which was subsequently centrifuged at $2500 \mathrm{rpm}$ for 10 minutes. The erythrocyte sediment was washed with physiological saline three times until the supernatant was no longer red ${ }^{38}$ in comparison to the color of normal saline. Erythrocyte pellets ( $1 \mathrm{~mL}$ ) were added to $49 \mathrm{~mL}$ of physiological saline to prepare a $2 \%$ erythrocyte standard suspension. The GL-BSA-HCPTNPs were dissolved in physiological saline at a concentration of $5 \mathrm{mg} / \mathrm{mL}$. Then, this solution $(0.125,0.25,0.5,1.0,1.5$, and $2 \mathrm{~mL}$ ) was added to six tubes, each containing $2.5 \mathrm{~mL}$ of the erythrocyte suspension. Physiological saline was added to each tube to a total volume of $5 \mathrm{~mL}$. Physiological saline ( $2.5 \mathrm{~mL}$ ) mixed with $2.5 \mathrm{~mL}$ of $2 \%$ erythrocyte suspension was used as negative control and the positive control was prepared by mixing $2.5 \mathrm{~mL}$ of water with $2.5 \mathrm{~mL} 2 \%$ erythrocyte suspension. After blending, all the tubes were incubated at $37^{\circ} \mathrm{C}$ and observed at baseline and after 1 hour, 2, 4, 7, 10, and 24 hours. Then the suspension in each tube was remixed lightly after 24 hours to observe agglutination of red cells.

\section{Targeting property assay}

\section{Cell culture}

Human hepatoma cells (SMMC7721) cells purchased for the Shanghai Cell Line Bank (Chinese Academy of Sciences, Shanghai, People's Republic of China) were used to perform the cell uptake study and cell proliferation assay of GL-BSA-HCPT NPs. The cells were cultured in RPMI 1640 medium with $10 \%$ heat-inactivated fetal bovine serum, penicillin $(100 \mathrm{U} / \mathrm{mL})$, and streptomycin $(100 \mu \mathrm{g} / \mathrm{mL})$, then incubated in a humidified atmosphere containing $\mathrm{CO}_{2}(5 \%)$ at $37^{\circ} \mathrm{C}$.

\section{Cell uptake}

The samples containing GL-BSA-HCPT-NPs, BSA-HCPTNPs, and the physical mixture of BSA-HCPT-NPs and GL were labeled with FITC in order to observe the extent of their uptake into SMMC7721 cells. The samples were dispersed in a carbonate buffer solution $(\mathrm{pH}=9)$. FITC was added to the dispersion at a ratio of 1:200. After blending, the reaction was performed by placing the samples in a dark place at $4^{\circ} \mathrm{C}$ for 2 hours. Following this, the FITC-labeled samples were separated by centrifugation at $12,000 \mathrm{rpm}$ for 20 minutes and the resultant supernatant discarded. The samples were then washed three times and separated again by centrifugation.

The cells (300,000 cells/well) were seeded in disposable six-well plates at $2 \mathrm{~mL} /$ well and cultured for 12 hours in a $5 \% \mathrm{CO}_{2}$ incubator at $37^{\circ} \mathrm{C}$. The FITC-labeled samples were added to different wells and incubated for 4 hours. After this, the wells were washed three times with normal saline, then the cells in each well were infiltrated with $1 \mathrm{~mL}$ saline. The plates were observed by laser scanning confocal microscopy (D-eclipse C1, Nikon, Tokyo, Japan) with an excitation wavelength of $488 \mathrm{~nm}$.

\section{Cell proliferation assay}

MTT assay was used to assess the cell proliferation of the SMMC7721 cells. The exponential growth-phase cells were seeded into disposable 96 -well plates at $200 \mu \mathrm{L} /$ well (containing 5000 cells) and cultured in a $5 \% \mathrm{CO}_{2}$ incubator at $37^{\circ} \mathrm{C}$ for 24 hours. The samples containing GL-BSAHCPT-NPs, BSA-HCPT-NPs, and HCPT sodium were added to wells at different concentrations of HCPT (248.0, 24.8, 2.48 , and $0.248 \mu \mathrm{g} / \mathrm{mL}$ ), and each concentration repeated five times. The cells were incubated for 48 hours. Following incubation, $20 \mu \mathrm{L}$ of MTT was added to each well, and the cells were incubated for another 4 hours. The supernatant was then discarded and $150 \mu \mathrm{L}$ dimethyl sulfoxide added to the wells. The 96-well plates were put on a horizontal oscillator to increase the solvation of formazan crystals. The optical density values were determined using a microplate reader at a wavelength of $492 \mathrm{~nm}$. The Bliss method ${ }^{39}$ was used to calculate the half maximal inhibitory concentration (IC50).

\section{Statistical study}

The data presented in the "Results and discussion" section are the mean \pm standard deviation of triplicate measurements and are representative of at least three independent experiments. 


\section{Results and discussion Experimental design and analysis}

The optimal conditions were determined using a singlefactor test. Particle size plays a key role in determining the distribution of nanoparticles (in this case, GL-BSAHCPT-NPs) in vivo and ensuring best access into cancer cells. During preliminary experiments, we determined that the following factors had a significant effect on particle size: BSA concentration, volume ratio of water to organic phase, and speed and speed duration of homogenization as well as pressure and number of times homogenized at each pressure (Table 1).

The first factor was BSA concentration. We selected a concentration range of $0.5-4 \mathrm{mg} / \mathrm{mL}$ to test. When determining the optimal BSA concentration, the other factors (ie, volume ratio of water to organic phase, speed and speed duration of homogenization, and pressure and pressure duration of homogenization) acted as fixed parameters. The particle size of $716.4 \pm 35.9 \mathrm{~nm}$ and $290 \pm 14.5 \mathrm{~nm}$ at the concentrations of $0.5 \mathrm{mg} / \mathrm{mL}$ and $4.0 \mathrm{mg} / \mathrm{mL}$, respectively, can be seen in Table 1. The results show that particle size decreased gradually as the BSA concentration was increased. BSA plays the role of surfactant in the system for containing both hydrophilic and hydrophobic groups. A better emulsified state is one where the organic phase and water phase mix uniformly, and the phenomenon of demulsification and stratification did not occur in this system within a certain time. However, according to Equation 1, when the concentration of HCPT is fixed, drug loading efficiency will be increased when the concentration of BSA is decreased. Taking this into account, we determined the concentration of $1 \mathrm{mg} / \mathrm{mL}$ to be optimum, and the experiments were studied at this concentration.

The ratio of water to organic phase volume was the second factor to be considered. We examined ratios within the range of 5:1 to 33:1. In determining the optimal ratio, the fixed parameters were BSA concentration $(1 \mathrm{mg} / \mathrm{mL})$, homogenizer speed and duration of speed. Particle size was $744.3 \pm 33.6 \mathrm{~nm}$ and $390.5 \pm 18.5 \mathrm{~nm}$ at the volume ratios of 5:1 and 9:1, respectively, as shown in Table 1. A water-in-oil emulsion system is formed at ratios between 5:1 and 9:1, with the particle size becoming smaller as the water volume is increased. However, we found that particle size visibly tends to increase in the ratio range of 9:1 to $17: 1$, but in this range, the water and organic phase cannot form a stable emulsion system. The same principle has been reported in the literature. ${ }^{22}$ There was a trend to smaller particle sizes and gradual stabilization of emulsion state at ratios from 17:1 to $33: 1$. The particle size when the water to organic phase volume ratio was $17: 1,21: 1$, and $25: 1$ were $842.1 \pm 41.5$, $787.2 \pm 33.9$, and $351.7 \pm 16.2 \mathrm{~nm}$, respectively, and at the ratio of $29: 1$ and 33:1, the particle sizes were $145.8 \pm 7.3$ and $115.7 \pm 5.6 \mathrm{~nm}$, respectively. Thus, we determined that a suitable ratio of oil to water would be in the range of 17:1 to 33:1 to ensure a stable emulsion state. Taking into account that the greater the volume of water used, the greater the amount of BSA is necessary, which would affect the drug loading efficiency, we selected the ratio of $25: 1$ as the optimal proportion of water to organic phase to use in subsequent tests.

The influence of homogenization speed and time on particle size is shown in Table 1. We tested homogenization speeds in the range of 5000-10,000 rpm and homogenization times of 1-12 minutes. The already established optimal BSA concentration $(1 \mathrm{mg} / \mathrm{mL})$ and ratio of water to organic phase volume (25:1) were used. At a speed of $5000 \mathrm{rpm}$, the resultant particle size was $55.1 \mathrm{~nm}$ after the first minute and this increased to $371.8 \pm 19.0 \mathrm{~nm}$ after 12 minutes. Over the initial 1-3 minutes, the particle size gradually became increased, but there was little change over the 5to 12-minute interval. Thus, it was determined that it was not useful to homogenize at this speed for a long period. Further, a longer homogenizing time may break the stability of the droplets, resulting in a larger particle size. At a speed of $7500 \mathrm{rpm}$, particle size was $290.5 \pm 14.1 \mathrm{~nm}$ after homogenization for 1 minute, and after 7 minutes particle size increased to $658.5 \pm 33.1 \mathrm{~nm}$. However, at 9 minutes, the size of the particle had decreased to $499.6 \pm 23.9 \mathrm{~nm}$, and it was $380.6 \pm 18.5 \mathrm{~nm}$ at 12 minutes. Therefore, we determined that at $7500 \mathrm{rpm}$, particle size becomes larger as homogenization time increases, but change in particle size amplitude is relatively small over the 3- to 12-minute period. At 10,000 rpm, particle sizes were $478 \pm 24.0 \mathrm{~nm}$ and $692 \pm 34.6 \mathrm{~nm}$ after homogenization for 1 minute and 12 minutes, respectively, and the change in particle size amplitude over time was the same as at $7500 \mathrm{rpm}$. Therefore, as product prepared at the speed of $5000 \mathrm{rpm}$ was of a smaller particle size than at other speeds (as shown in Table 1), we determined that $5000 \mathrm{rpm}$ was powerful enough for our experiments. Higher speeds were not helpful, as they negatively influenced the goal of reaching particles of a smaller size. Therefore, a speed of $5000 \mathrm{rpm}$ for a duration of 1 minute was chosen as being optimal.

Primary emulsification could occur under the three optimal conditions just described - a BSA concentration of $1 \mathrm{mg} / \mathrm{mL}$, a ratio of $25: 1$ of water to organic phase volume, and a homogenization speed of $5000 \mathrm{rpm}$ for 1 minute. 
The system under these conditions was observed by optical microscope and the optimal emulsified state was observed, with many small droplets of oil in water, as shown in Figure 2. Further, abundant droplets dispersed in the solvent were seen under magnification of $10 \times 40$ times (Figure $2 \mathrm{~A}$ ) and under magnification of $10 \times 100$ times (Figure $2 \mathrm{~B}$ ). The droplets observed were in good condition. This optimal emulsified state after homogenization has also been reported in the literature. ${ }^{40}$

Homogenization can ensure a smaller particle size and a more uniform droplet. ${ }^{41}$ As heat can induce the crosslinking of BSA when BSA is heated for the homogenization process, cross-linking occurs, which helps to form stable NPs. ${ }^{42}$ A sample was prepared under the optimal conditions just described to study the impact of homogenization pressure and the number of homogenizations at each pressure on particle size (Table 1). We tested homogenization pressures in the range of 400-1200 bar, homogenized between three and 15 times. Cross-linking of BSA did not occur in the system as soon as it became homogeneous, but began on subsequent homogenizations; as the number of times homogenization was performed increased, the temperature of the system increased. In conclusion, we determined that the greater the homogenization pressure, the sooner stable nanoemulsion droplets would be obtained. At a pressure of 400 bar, the particle size remained stable when homogenization was repeated three and eleven times (Table 1). When homogenized 13 times, the system started to become transparent and opalescent, indicating that the BSA had cross-linked; at this point, the particle size was $237 \pm 10.3 \mathrm{~nm}$. After 15 homogenizations, we observed a trend of decreasing particle size, due to the continuous homogenization. At a pressure of 600 bar, a good emulsified state was observed after eleven homogenizations, at which point the particle size was $173.4 \pm 6.2 \mathrm{~nm}$. After 13 and 15 homogenizations, the particle sizes were $233.1 \pm 13.5$ and $368.7 \pm 14.9 \mathrm{~nm}$, respectively. At a pressure of 800 bar, a good emulsified
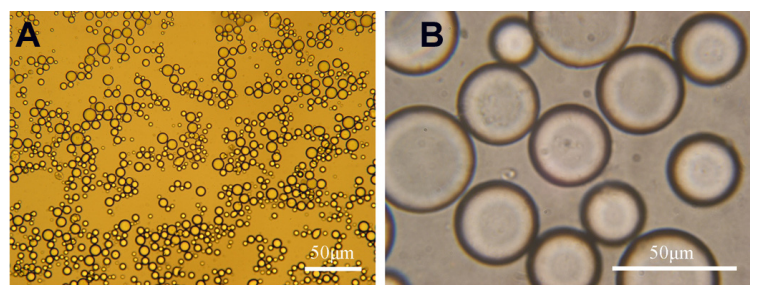

Figure 2 The state of the system after homogenization observed by optical microscope: $($ A) $10 \times 40$ magnification; (B) $10 \times 100$ magnification. state occurred after seven homogenizations, which resulted in a particle size of $144.3 \pm 6.1 \mathrm{~nm}$. Particle size increased significantly from 9 homogenizations and changed slightly from 9 to 15 homogenizations. A good state of emulsification occurred after five homogenization at pressures of 1000 and 1200 bar, resulting in particle sizes of $225.9 \pm 11.2$ and $221.2 \pm 11.2 \mathrm{~nm}$, respectively. As the number of homogenizations increased, the particle size gradually increased. Even when the pressure was different, the particle size of the product continued increase with subsequent homogenizations following achievement of a good emulsified state, which we attributed to the continual cross-linking of the BSA at high temperature. It is favorable to choose the number of homogenizations to match the primary period of BSA cross-linking. Particle size was smallest at 800 bar, as shown in Table 1. Therefore, a pressure of 800 bar and seven homogenizations were selected as optimal.

HCPT solubility was significantly improved when the organic phase consisted of trichloromethane and ethanol rather than water; however, the level of solubility was still not as good as hoped. The peak concentration of HCPT $(3.3 \mathrm{mg} / \mathrm{mL})$ was selected directly in the preparation process.

In sum, the optimal conditions for the production of GLBSA-HCPT-NPs were: the peak concentration of HCPT, BSA concentration of $1 \mathrm{mg} / \mathrm{mL}$, ratio of water to organic phase of 25:1, and seven homogenizations at a speed of 5000 rpm for 1 minute each at a pressure of 800 bar.

HCPT-loaded BSA has been studied and reported in the literature. Yang et al reported the preparation of HCPT-loaded BSA-NPs using an emulsification method in $2007 .{ }^{21}$ In that study, mean particle size of the obtained NPs was around $600 \mathrm{~nm}$, and the drug loading contents, encapsulation efficiencies, and yields achieved were $2.21 \%, 57.5 \%$, and $90.5 \%$, respectively, under optimal preparation conditions. ${ }^{21}$ Further, Li et al reported the preparation of HCPT-loaded BSA-NPs in 2011 using an improved preparation process. The particle size of the NPs obtained was $233.9 \pm 1.2 \mathrm{~nm}$ and they achieved a drug loading content and encapsulating efficiency of $7.3 \%$ and $79.1 \%$, respectively. ${ }^{17}$ The preparation process detailed in the present paper achieved greater progress with not only particle size but also drug loading efficiency and drug entrapment efficiency. The GL-BSA-HCPT-NPs prepared under the determined optimal conditions had a particle size of $157.5 \mathrm{~nm}$ and zeta potential of $-22.51 \pm 0.78 \mathrm{mV}$. Further, the drug loading efficiency and drug entrapment efficiency were $10.9 \%$ and $93.7 \%$, respectively, and these were used for the detection of the following. 


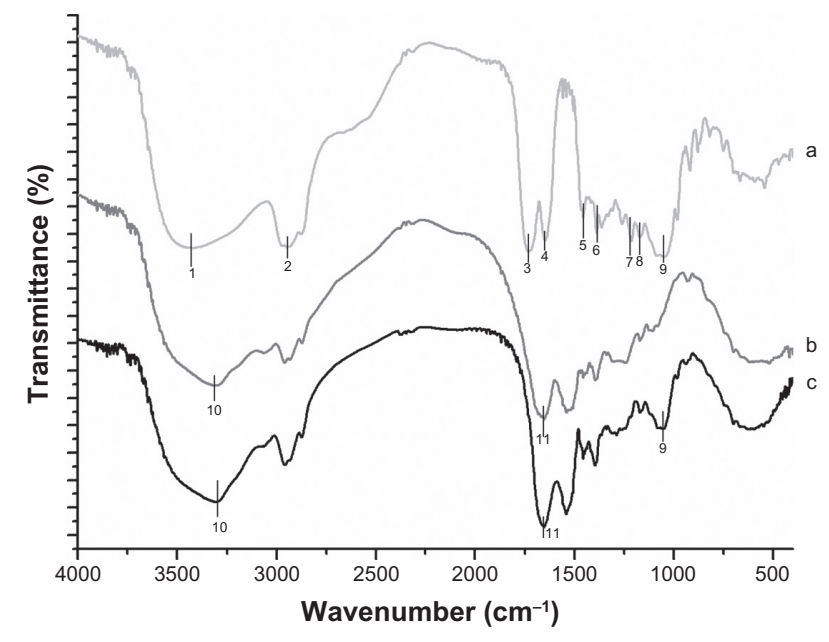

Figure 3 Fourier transform infrared spectra of samples: (a) raw glycyrrhizic acid, (b) raw bovine serum albumin, (c) glycyrrhizic acid-conjugated bovine serum albumin. Notes: The peaks correspond to: (I) $3431.36 \mathrm{~cm}^{-1}$, FreeOH; (2) $2953.45 \mathrm{~cm}^{-1}, \mathrm{C}-\mathrm{CH} 3$; (3) $1731.38 \mathrm{~cm}^{-1}, \mathrm{C}=\mathrm{O}$; (4) $1647.21 \mathrm{~cm}^{-1}, \mathrm{C}=\mathrm{O}$; (5) $1454.33 \mathrm{~cm}^{-1}, \mathrm{CH} 2$; (6) $1388.75 \mathrm{~cm}^{-1}, \mathrm{CH} 3$; (7) $1215.15 \mathrm{~cm}^{-1}$, acetates; (8) $1172.72 \mathrm{~cm}^{-1}$, formates; (9) $1052.90 \mathrm{~cm}^{-1}$, secondary cyclic alcohols; (10) $3307.92 \mathrm{~cm}^{-1}$, free $\mathrm{NH}$; (II) $1656.78 \mathrm{~cm}^{-1}$, amides.

\section{The coupling degree of GL to BSA}

The FTIR spectra of samples are presented in Figure 3, which shows the remarkable peaks of BSA (peaks 10 and 11) and GL (peaks 1-9). The FTIR spectrum of GL-BSA (c) shows both the characteristic peak of GL (peak 9) at wavenumber $1052.90 \mathrm{~cm}^{-1}$ and of BSA (peak 10) at $3307.92 \mathrm{~cm}^{-1}$. As BSA contains a large number of amide bonds, there is an absorption peak at wavenumber $1656.78 \mathrm{~cm}^{-1}$. The curve $\mathrm{c}$ also shows peak 11, which is more obvious than in curve b, as the completion of GL conjugation to BSA depends on the formation of amide bonds. Thus, the spectra show that GL was coupled with BSA successfully.

GL, the active compound in licorice, is a conjugate of two molecules - glucuronic acid and GA. ${ }^{43}$ GL is turned into GA by acid hydrolysis, and this was used to detect the quantity of GL coupled to BSA, which, using HPLC, was found to be $98.26 \mu \mathrm{g} / \mathrm{mg}$.

\section{Characterization of GL-BSA-HCPT-NPs Morphology of GL-BSA-HCPT-NPs}

The morphology of the samples is shown in Figure 4. The raw BSA appeared as irregular flakes, with a particle size ranging from 1 to $200 \mu \mathrm{m}$ (Figure 4A). Raw HCPT presented as irregular blocks, with a particle size ranging from 1 to $20 \mu \mathrm{m}$ (Figure 4B). Figure 4C shows BSA-NPs with a nearly spherical shape and Figure 4D shows GLBSA-NPs with a nearly spherical shape. The GL-BSAHCPT-NPs were nearly spherical and dispersed evenly, rather than stuck together, as can be seen in Figure 4E.
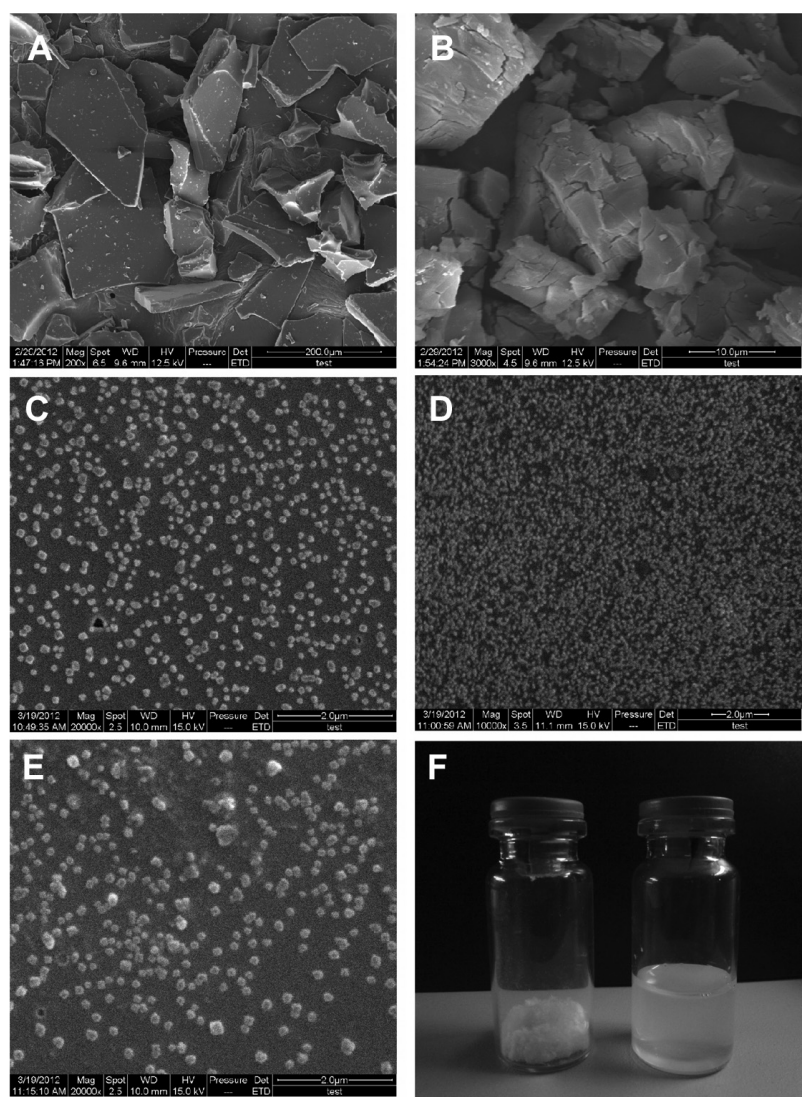

Figure 4 Morphology of samples observed by scanning electron microscopy: (A) raw bovine serum albumin (BSA), (B) raw 10-hydroxycamptothecin (HCPT), (C) BSA-nanoparticles (NPs), (D) glycyrrhizic acid-conjugated BSA-NPs, and (E) I0-hydroxycamptothecin-loaded glycyrrhizic acid-conjugated bovine serum albumin nanoparticles (GL-BSA-HCPT-NPs). (F) Photograph of freeze-dried GLBSA-HCPT-NP powder (left) and the aqueous dispersion (right).

The freeze-dried powder and aqueous dispersion of the powder are shown in Figure 4F. In this photograph, it can be seen that the freeze-dried powder reconstitutes well in solution, with a transparent opalescence and uniform state obeservable.

\section{In vitro stability test}

The dispersion of GL-BSA-HCPT-NPs in water was studied to measure the stability of the solution after 16 hours at a temperature of $25^{\circ} \mathrm{C}$. The results of this test are shown in Table 2. The particle size was found to be always $<200 \mathrm{~nm}$ and zeta potential was in the range of $-20.34 \pm 4.60 \mathrm{mV}$ to $-36.83 \pm 2.25 \mathrm{mV}$. The polydispersity index detected at baseline was 0.164 , and it was stable in the range of $0.251-0.307$. There was a limited amount of NP aggregation during the first hour, but the dispersion was subsequently stable and uniform for the rest of the observation period. The phenomena of particle reunion, precipitation, and stratification did not occur when the solution was stored after 16 hours and the dispersion appeared equally 
Table 2 Stability study of 10-hydroxycamptothecin-loaded glycyrrhizic acid-conjugated bovine serum albumin nanoparticles examining particle size and zeta potential changes over 16 hours

\begin{tabular}{llll}
\hline $\begin{array}{l}\text { Time } \\
(\mathbf{h})\end{array}$ & $\begin{array}{l}\text { Particle size } \pm \text { SD } \\
(\mathbf{n m})\end{array}$ & $\begin{array}{l}\text { Zeta potential } \pm \text { SD } \\
(\mathbf{m V})\end{array}$ & $\begin{array}{l}\text { Polydispersity } \\
\text { index }\end{array}$ \\
\hline 0 & $168.5 \pm 8.1$ & $-27.72 \pm 1.76$ & 0.164 \\
1 & $122.0 \pm 1.6$ & $-28.91 \pm 2.44$ & 0.307 \\
2 & $118.5 \pm 4.2$ & $-29.00 \pm 1.91$ & 0.305 \\
3 & $116.1 \pm 4.1$ & $-25.78 \pm 1.32$ & 0.300 \\
4 & $131.2 \pm 9.7$ & $-23.72 \pm 2.53$ & 0.280 \\
5 & $132.8 \pm 7.8$ & $-23.57 \pm 1.53$ & 0.260 \\
6 & $127.6 \pm 7.2$ & $-27.99 \pm 2.03$ & 0.279 \\
7 & $139.8 \pm 7.6$ & $-30.20 \pm 3.33$ & 0.242 \\
8 & $133.0 \pm 11.8$ & $-33.07 \pm 2.81$ & 0.251 \\
9 & $122.6 \pm 15.0$ & $-35.36 \pm 2.47$ & 0.269 \\
10 & $146.5 \pm 5.3$ & $-36.02 \pm 3.05$ & 0.232 \\
11 & $\mid 33.9 \pm 2.4$ & $-36.83 \pm 2.25$ & 0.260 \\
12 & $\mid 24.3 \pm 3.4$ & $-20.34 \pm 4.60$ & 0.283 \\
13 & $\mid 18.1 \pm 2.8$ & $-28.95 \pm 1.28$ & 0.299 \\
14 & $\mid 33.6 \pm 6.4$ & $-26.15 \pm 1.29$ & 0.261 \\
15 & $\mid 23.7 \pm 5.0$ & $-21.75 \pm 1.34$ & 0.292 \\
16 & $\mid 19.0 \pm 3.1$ & $-24.16 \pm 1.7 \mid$ & 0.303 \\
\hline
\end{tabular}

Abbreviation: SD, standard deviation.

distributed. Therefore, the GL-BSA-HCPT-NP dispersion demonstrated good stability over the 16-hour observation period.

\section{Solid state study}

The FTIR spectra of samples is shown in Figure 5A. As can be seen in this figure, the GL-BSA and GL-BSA-HCPT-NP curves are basically consistent. The distinctive HCPT peak appears in the raw HCPT at wavenumber $1741.33 \mathrm{~cm}^{-1}$ and there is also a small peak in the curve of the GL-BSA-HCPTNPs, which indicates that there was a trace amount of HCPT present on the surface of the GL-BSA.

TG measures the relationship between temperature and quality. The TG curves of samples are shown in Figure 5B. The HCPT began to lose weight from $20^{\circ} \mathrm{C}$ to $80^{\circ} \mathrm{C}$, which was attributed to water loss. The weight loss of HCPT happened again from about $300^{\circ} \mathrm{C}$. The total ratio of weight loss is about $55 \%$. The curves of raw BSA, GL-BSA, and the GLBSA-HCPT-NPs show weight loss from $20^{\circ} \mathrm{C}$ to $100^{\circ} \mathrm{C}$ due to water loss. The phenomenon of weight loss happened again and the weight decreased rapidly from about $250^{\circ} \mathrm{C}$. The raw BSA lost about $67 \%$ of its weight, as can be seen in the b curve in Figure 5A. The highest weight loss - 91\% - was observed in GL-BSA-NPs. This is because the GL-BSA-NPs have a smaller particle size than raw BSA, and the smaller particle size has a higher specific surface area and hence higher specific surface energy, ${ }^{44}$ which leads to easier vaporization and earlier decom- position energy. The weight loss rate for GL-BSA-HCPT-NPs was about $80 \%$, which was lower than that for GL-BSA and higher than that for HCPT, which effectively confirmed the presence of HCPT in the NPs.

The XRD analysis of samples containing raw HCPT, raw BSA, GL-BSA-NPs, and GL-BSA-HCPT-NPs is shown in Figure 5C. Many diffraction peaks can be observed in the XRD curve of raw HCPT due to its crystal morphology, ${ }^{17}$ while there are two broad peaks (at $2 \theta=8.23^{\circ}$ and $21.59^{\circ}$ ) in the curve of BSA on account of its amorphous morphology. The GL-BSA-NP curve has only one broad diffraction peak (at $2 \theta=22.6^{\circ}$ ) and the NPs were confirmed as amorphous because of their smaller particle size. Only one broad peak was found in the XRD curve of GL-BSA-HCPT-NPs, showing that HCPT is wrapped and amorphous, and that there was a small amount of HCPT present on the surface of GL-BSA, which is in agreement with the FTIR spectroscopy result (Figure 5A). An amorphous solid is more diffluent than a crystalline solid; ${ }^{45}$ as a result, GL-BSA-HCPT-NPs have good solubility in water compared with raw HCPT.

The DSC curves of samples are shown in Figure 5D. There is an obvious melting peak at about $278.9^{\circ} \mathrm{C}$ in the HCPT curve, but no melting peak in either the BSA or GL-BSA curves, confirming that both were amorphous. Moreover, there is no melting peak for HCPT in GL-BSAHCPT-NPs, as shown in Figure 5D, which confirmed the presence of the amorphous HCPT in the GL-BSA-HCPTNPs, in accordance with the XRD results.

\section{Drug-release study}

HCPT sodium injection and $10.9 \%$ drug loading efficiency of GL-BSA-HCPT-NPs were both used to study the release of HCPT in vitro (Figure 6). The release of GL-BSAHCPT-NPs is shown in curve $b$ in this figure. It was nearly linear in the first 10 hours, and the speed of release was constant. The accumulated release of GL-BSA-HCPTNPs was $43.25 \%$. The particle size and zeta potential of GL-BSA-HCPT-NPs dispersion was relatively stable, as shown in Table 2. Studies on drug release from BSA have been reported in the literature, ${ }^{46}$ and have indicated that BSA degradation is impossible in the first hour. The particles did not disperse completely in water at baseline, but began to disperse completely from 1 hour on, maintaining a stable particle size over time (Table 2). At 12 hours, the rate of GL-BSA-HCPT-NP release increased rapidly. The total accumulated release was $75.42 \%$. This phenomenon may be due to the degradation of BSA. The particle size of 
A

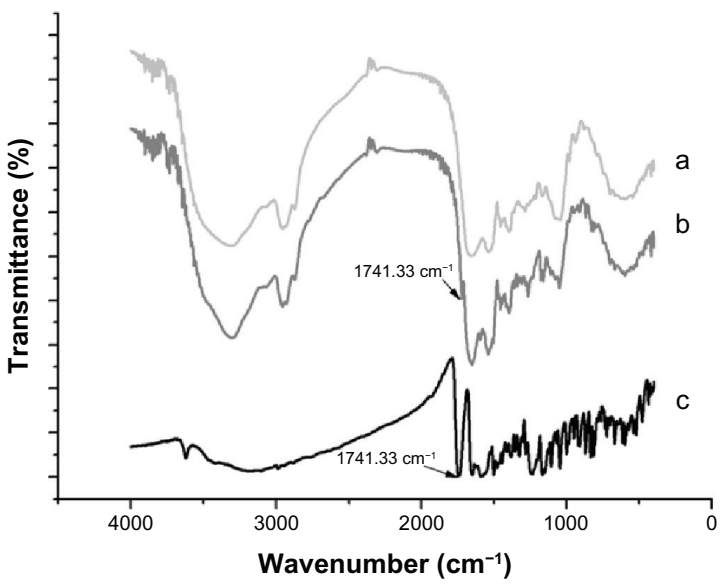

C

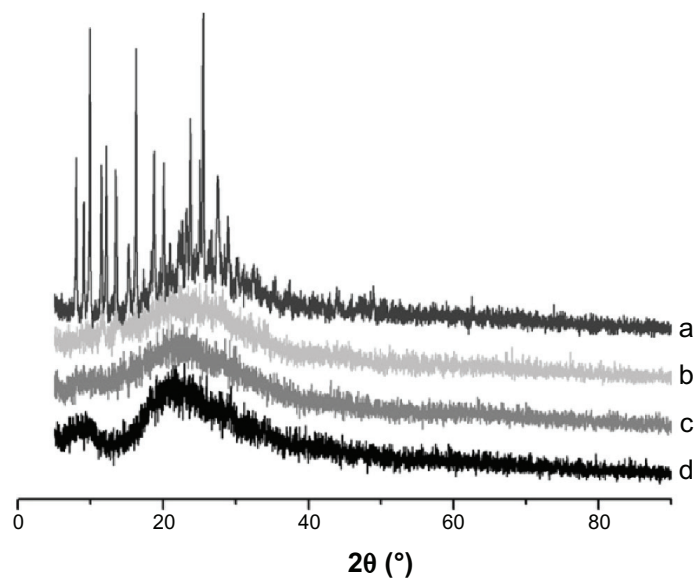

B

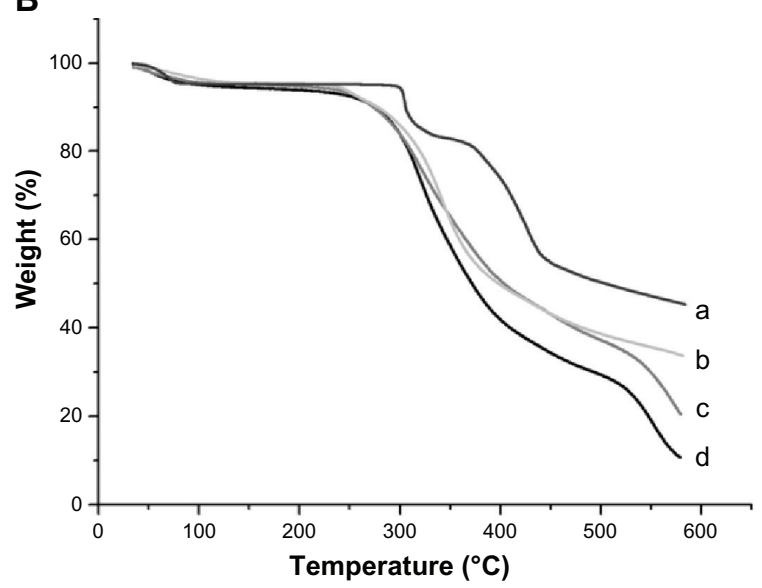

D

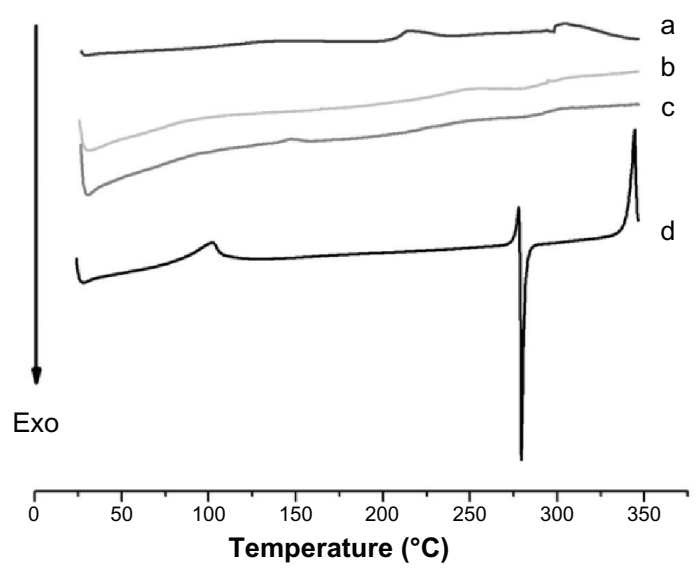

Figure 5 Physical properties of samples. (A) Fourier transform infrared spectra of samples: (a) glycyrrhizic acid-conjugated bovine serum albumin (GL-BSA), (b) 10-hydroxycamptothecin-loaded glycyrrhizic acid-conjugated bovine serum albumin nanoparticles (GL-BSA-HCPT-NPs), and (c) raw I0-hydroxycamptothecin (HCPT). (B) Thermogravimetric curves of samples: (a) raw HCPT, (b) raw BSA, (c) GL-BSA-HCPT-NPs, and (d) glycyrrhizic acid-conjugated bovine serum albumin nanoparticles (GL-BSA-NPs). (C) X-ray diffraction curves of samples: (a) raw HCPT, (b) GL-BSA-HCPT-NPs, (c) GL-BSA-NPs, and (d) raw BSA. (D) Differential scanning calorimetric curves of samples: (a) raw BSA, (b) GL-BSA-NPs, (c) GL-BSA-HCPT-NPs, and (d) raw HCPT.

the GL-BSA-HCPT-NP dispersion was smaller at 12 hours than at 10 hours, and the zeta potential rose over this period (Table 2). The results show that plenty of HCPT was released from the NPs.

The GL-BSA-HCPT-NPs continued to be released slowly over the following 60 hours, with cumulative release reaching $\sim 87 \%$ after 72 hours. At 10 hours, the accumulated release of sodium was $82.72 \%$, nearly twice the accumulated release of GL-BSA-HCPT-NPs at the same time. At 12 hours, the accumulated release of sodium had reached $84.85 \%$, but the rate of release gradually leveled off and the cumulative release had reached $\sim 96 \%$ at 72 hours.

The release of another positive control (HCPT sodium solution with GL-BSA in a physical mixture) is shown as curve c in Figure 6. It can be seen that the rate of mixture release was more rapid than for the other two samples. At 10 hours, the cumulative release was $94.35 \%$. At 12 hours, the cumulative release rate was $84.85 \%$ for HCPT sodium and $96.12 \%$ for the HCPT sodium/GL-BSA mixture. Following this, the HCPT sodium/GL-BSA mixture was released slowly, to reach a total cumulative release of $98.14 \%$ at 72 hours. It is clear that the HCPT sodium/GL-BSA mixture was released more rapidly than HCPT sodium alone. This was due to the influence of GL-BSA in the mixture, which acts as a surfactant, helping to improve the solubility and speed of solubility of the HCPT sodium in the mixture. These results indicate that the drug delivery system can release continuously and slowly.

\section{Hemolysis test}

Hemolysis only occurred in the positive control tube, shown in Figure 7. Tube 1 was the negative control, Tubes 2-7 contained samples at different concentrations of GL-BSA-HCPT- 


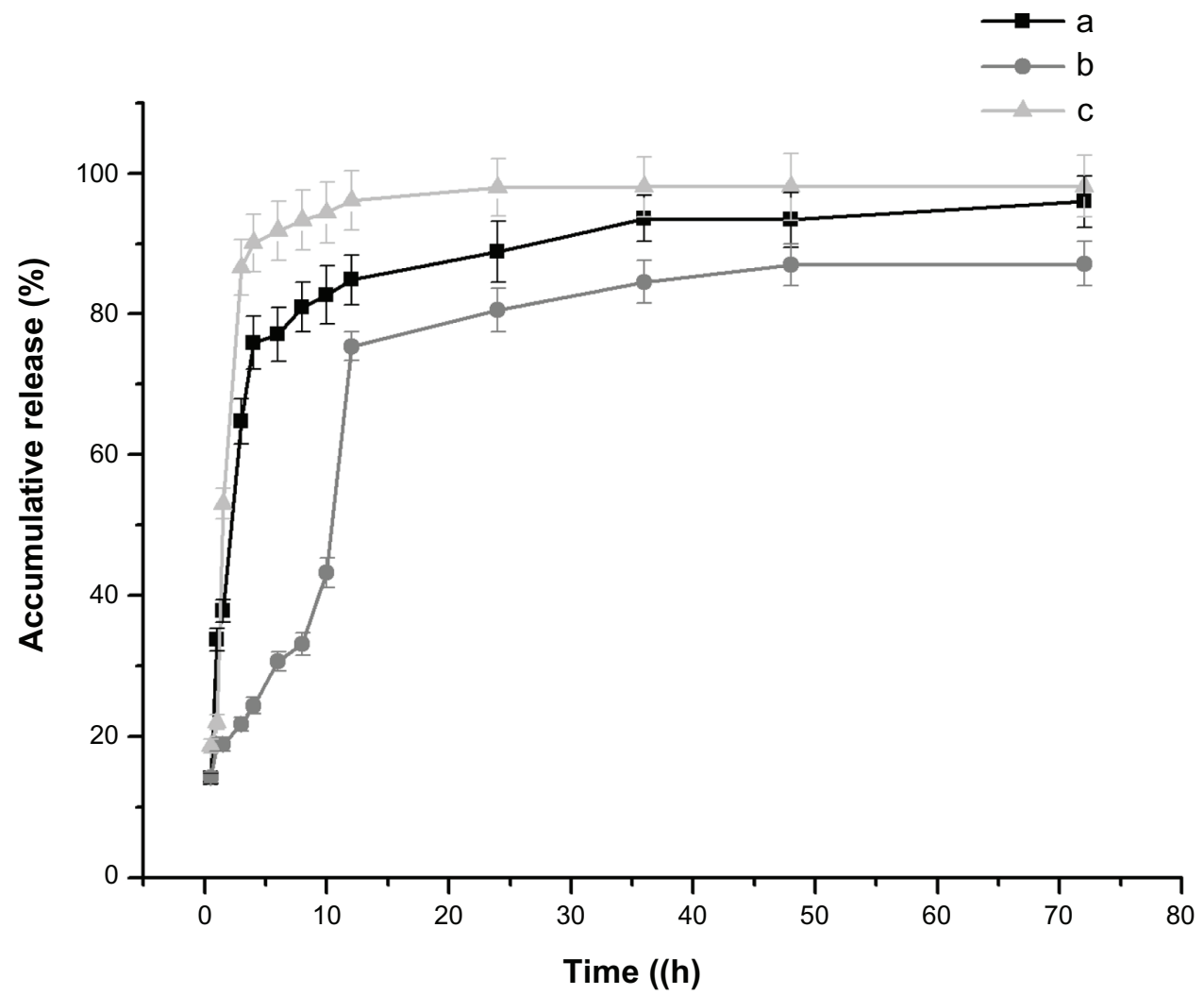

Figure 6 Drug-release curves: (a) 10-hydroxycamptothecin (HCPT) sodium injection, (b) HCPT-loaded glycyrrhizic acid (GL)-conjugated bovine serum albumin (BSA) nanoparticles, (c) HCPT sodium solution with GL-conjugated BSA in physical mixture.

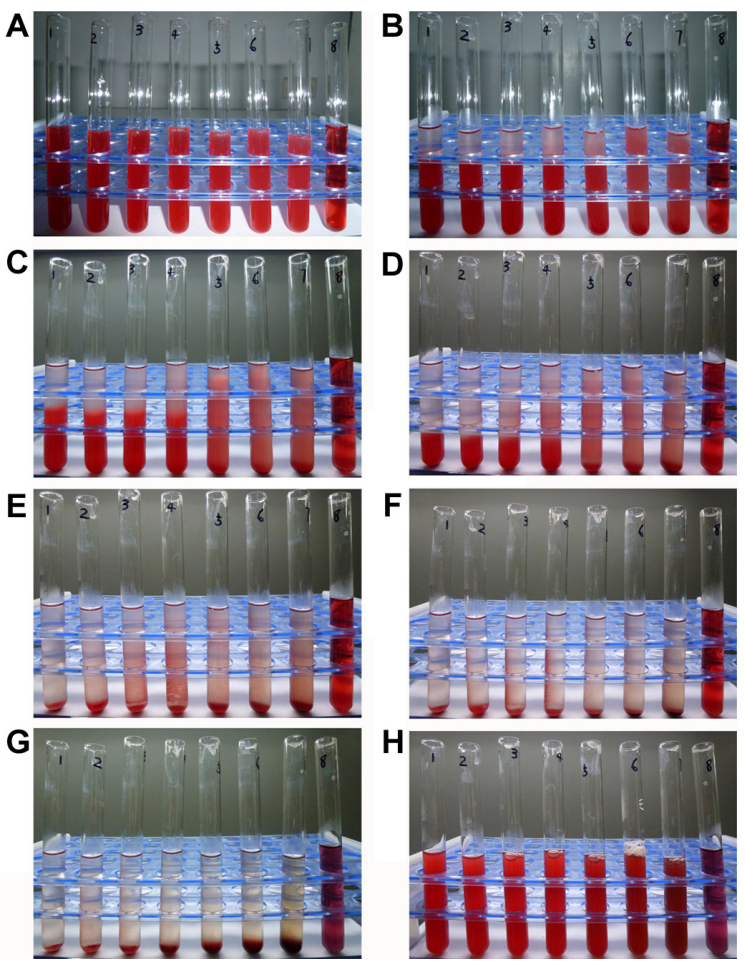

Figure 7 Hemolysis testing of 10-hydroxycamptothecin-loaded glycyrrhizic acidconjugated bovine serum albumin nanoparticles from 0 to 24 hours: (A) 0 hours, (B) I hour, (C) 2 hours, (D) 4 hours, (E) 7 hours, (F) 10 hours, (G) 24 hours, (H) 24 hours (well shaken).
NPs, and Tube 8 was the positive control. After re-mixing, red blood cells were uniformly distributed in Tubes $2-7$ without erythrocyte agglutination, and consistent with the negative control. The highest drug concentration was $2 \mathrm{mg} / \mathrm{mL}$ in Tube 7, in which there was no occurrence of hemolysis and agglutination at $37^{\circ} \mathrm{C}$. Thus, GL-BSA is a safe drug carrier for tumor-targeted drug delivery. Lian et al have also proven the safety of drug carriers in this way. ${ }^{38}$

\section{Targeting property assay}

\section{Cell uptake}

The fluorescence intensity of the FITC-labeled samples is shown in Figure 8. The fluorescence intensity of FITClabeled GL-BSA-HCPT-NPs was more obvious than that of the other samples, due to the abundant GL-BSA-HCPTNPs captured and adsorbed by the cells (Figure 8A). The well containing the physical mixture of FITC-labeled BSA-HCPT-NPs and GL showed some fluorescence spots (Figure 8B), but the intensity was obviously weaker than the fluorescence shown in Figure 8A and C. We proposed that the main reason for the reduced BSA-HCPT-NP uptake was that the SMMC7721 cells can selectively capture GL. Figure $8 \mathrm{C}$ confirms this inference, as there are more 

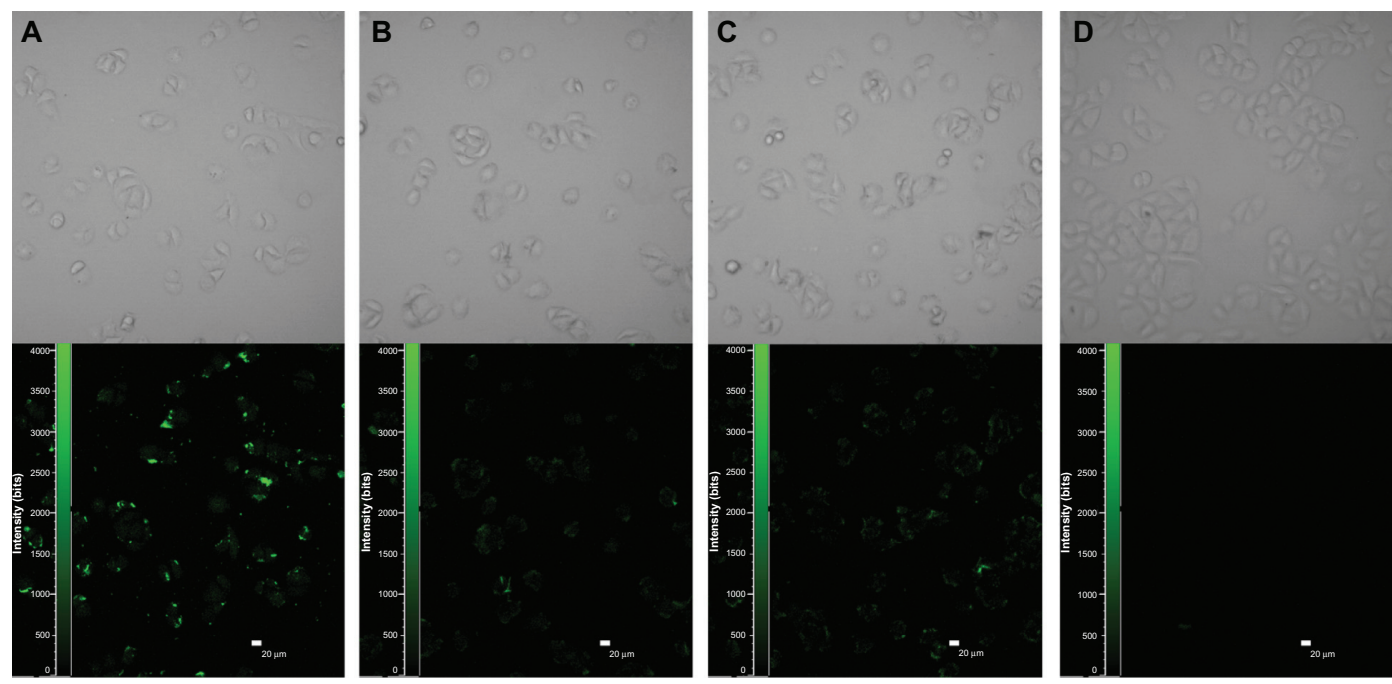

Figure 8 The fluorescence intensity of samples using fluorescein isothiocyanate (FITC) labeling. (A) Laser scanning confocal microscope (LSCM) image of 10-hydroxycamptothecin-loaded glycyrrhizic acid-conjugated bovine serum albumin nanoparticles (GL-BSA-HCPT-NPs) (top) and FITC-labeled GL-BSA-HCPT-NPs (bottom); (B) LSCM image of the physical mixture of I0-hydroxycamptothecin-loaded bovine serum albumin nanoparticles (BSA-HCPT-NPs) and GL (top) and FITC-labeled BSAHCPT-NPs and GL (bottom); (C) LSCM image of BSA-HCPT-NPs (top) and FITC-labeled BSA-HCPT-NPs; (D) LSCM image of blank cells to which no NPs were added. Abbreviations: BSA, bovine serum albumin; GL, glycyrrhizic acid; HCPT, I0-hydroxycamptothecin; NP, nanoparticle.

fluorescence spots in this figure than in Figure 8B. Figure $8 \mathrm{C}$ shows the fluorescence intensity of FITC-labeled BSA-HCPT-NPs is weaker than in Figure 8A, but a little stronger than in Figure 8B. Figure 8D shows almost no fluorescence spots, as there were no NPs in this well. Thus, the fluorescence intensity of the samples was such that Figure $8 \mathrm{~A}>8 \mathrm{C}>8 \mathrm{~B}>8 \mathrm{D}$. As GL is mainly taken up into the liver, ${ }^{30} \mathrm{GL}$-conjugated NPs display a better rate of cell uptake than unconjugated NPs.

\section{Cell proliferation assay}

MTT assay was used to study the cell proliferation of SMMC7721 cells. This method was selected because it is a classical procedure that has been mentioned many times in the literature. ${ }^{47,48}$ The inhibitory rates of the samples are shown in Figure 9. The concentrations of GL-BSAHCPT-NPs tested were 248.0, 24.8, 2.48, and 0.248 $\mu \mathrm{g} / \mathrm{mL}$. Inhibition of cell proliferation occurred after incubation for 48 hours, and developed alongside rising concentration. Figure 9A shows the inhibitory rate of HCPT sodium, which is lower than that displayed in Figure 9B and $\mathrm{C}$ for each drug concentration. Figure 9B shows the inhibition of BSA-HCPT-NPs and Figure 9C shows the inhibitory rate of GL-BSA-HCPT-NPs. GL-BSA-HCPT-NPs could inhibit cell proliferation more effectively than BSA-HCPT-NPs and HCPT sodium, and this was especially true at low concentration. The high inhibitory rates of GL-BSA-HCPTNPs and BSA-HCPT-NPs show the superiority of HCPT over any HCPT sodium salt.
The NPs were captured by cell endocytosis. Degradation of BSA led to the release of HCPT from NPs captured in the cells, which inhibits the cell proliferation. Owing to the livertaxis of GL, GL-BSA-HCPT-NPs were taken up in larger number by cells and appeared more efficacious than BSA-HCPT-NPs. The IC50s of GL-BSA-HCPT-NPs, BSA-HCPT-NPs, and HCPT sodium were $0.78 \pm 0.015$, $1.62 \pm 0.039$, and $7.93 \pm 0.255 \mu \mathrm{g} / \mathrm{mL}$, respectively. A lower concentration of BSA-HCPT-NPs was required to reach the inhibition ratio of $50 \%$ after incubation for 48 hours than of HCPT sodium, which shows the superiority of NPs and an efficacy limitation of HCPT sodium. The lowest concentration required to reach the $50 \%$ inhibition ratio after 48 hours was of GL-BSA-HCPT-NPs, which shows that NPs conjugating GL are more efficacious than non-conjugated NPs.

Based on the results of the cell uptake and cell proliferation assays, we predict the superiority of GL-BSA-HCPT-NPs over BSA-HCPT-NPs and HCPT sodium in future in vivo studies. As the liver can take up GL specifically, GL would function as a guide for GL-BSA-HCPT-NPs, drawing them to the liver cancer cells or tissue, thus improving liver uptake. In vivo studies on tumor models will be carried out in future. Figure 10 shows the principle of NP uptake by the liver.

\section{Conclusion}

In the study, GL-BSA-HCPT-NPs were prepared with a narrow particle size distribution and an average particle size of $157.5 \mathrm{~nm}$ by high-pressure homogenization 


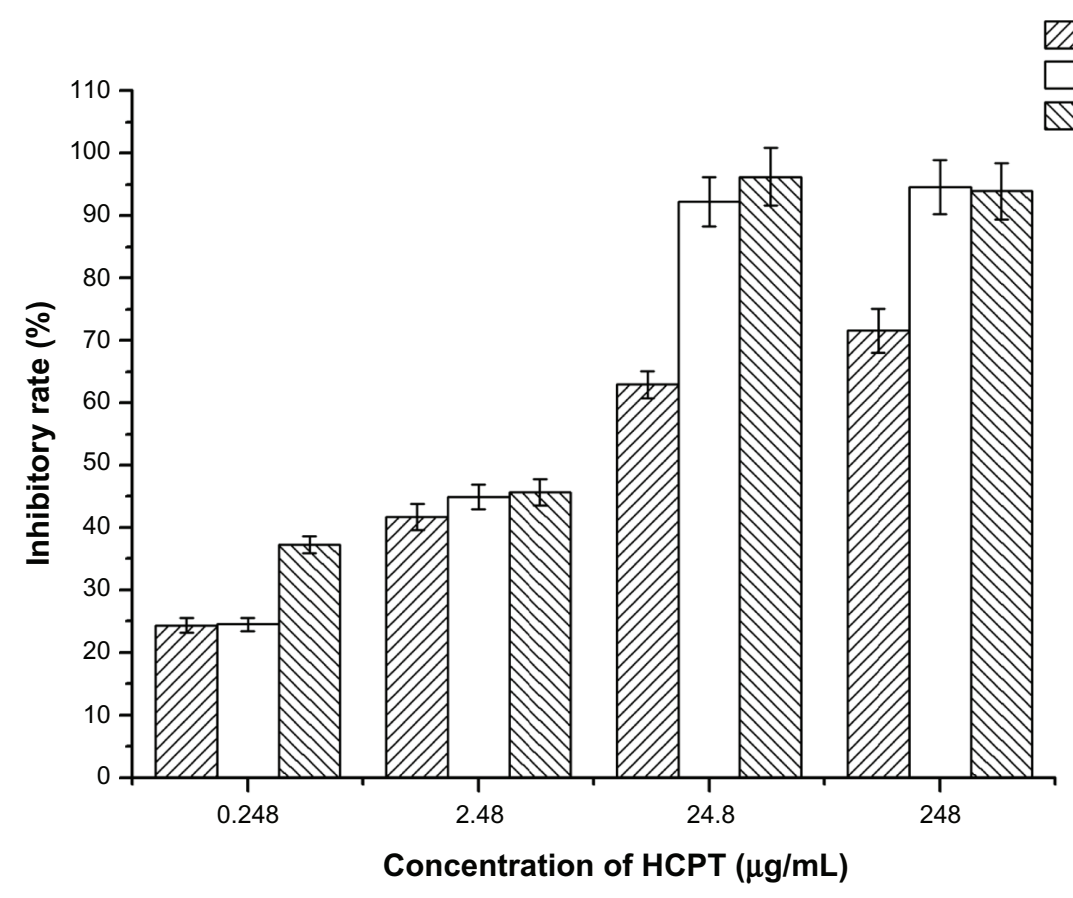

Figure 9 Inhibitory rates of samples: (A) 10-hydroxycamptothecin (HCPT) sodium, (B) 10-hydroxycamptothecin-loaded bovine serum albumin nanoparticles, and (C) 10-hydroxycamptothecin-loaded glycyrrhizic acid-conjugated bovine serum albumin nanoparticles.
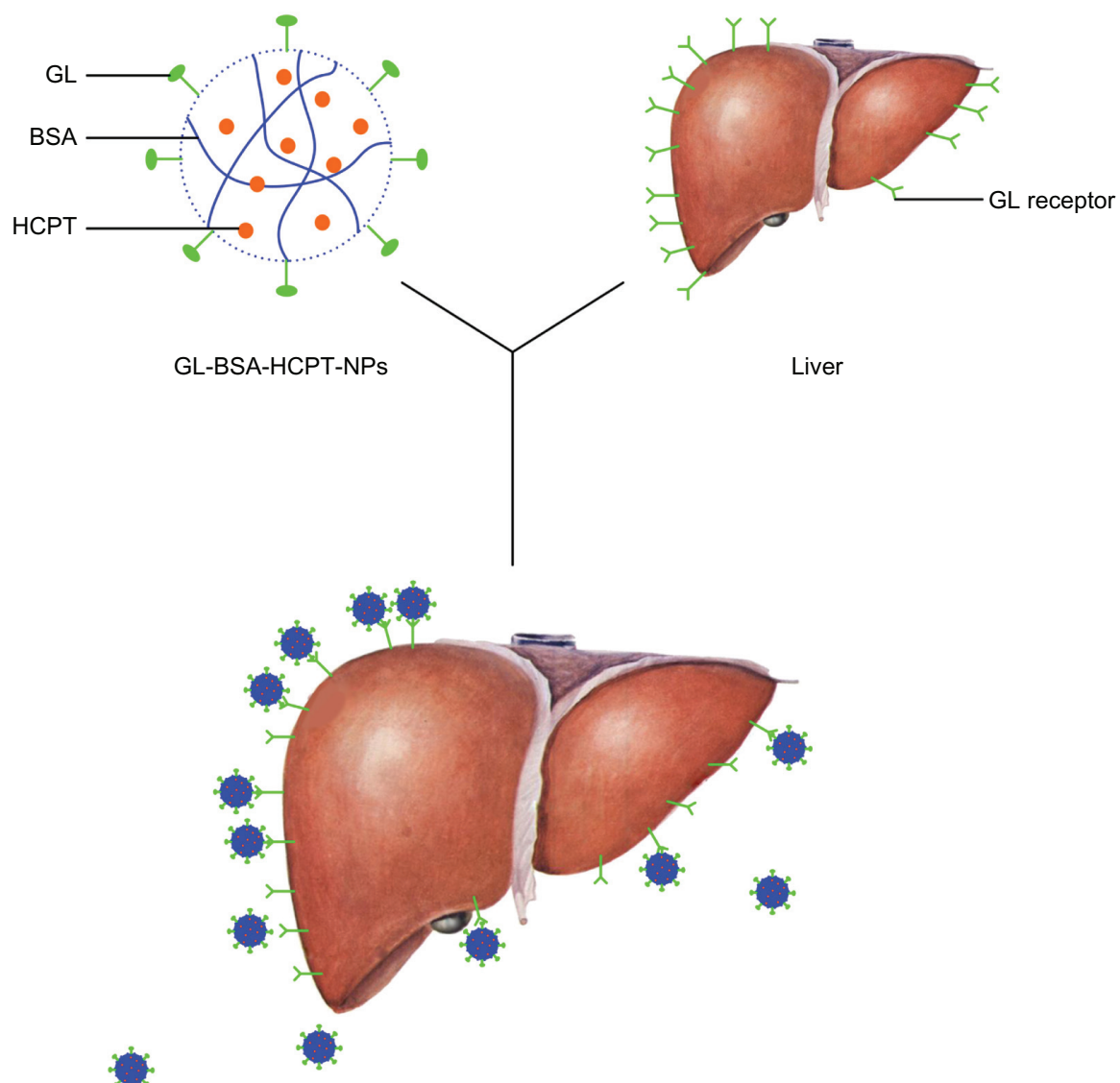

Figure 10 Diagram showing how the liver may interact with 10-hydroxycamptothecin-loaded glycyrrhizic acid-conjugated bovine serum albumin nanoparticles (GL-BSAHCPT-NPs).

Abbreviations: BSA, bovine serum albumin; GL, glycyrrhizic acid; HCPT, 10-hydroxycamptothecin. 
emulsification. The zeta potential of the GL-BSA-HCPTNPs was $-22.51 \pm 0.78 \mathrm{mV}$, indicating stablity for storage. The aqueous solution of GL-BSA-HCPT-NPs was stable within 16 hours, as shown in our stability test. The drug entrapment efficiency and drug loading efficiency were $93.7 \%$ and $10.9 \%$, respectively. In addition, the characteristics of the GL-BSA-HCPT-NPs were determined by SEM, FTIR spectroscopy, TG, XRD, and DSC analyses. The results show that the GL-BSA-HCPT-NPs were nearly spherical in shape and HCPT presented amorphously wrapped in GL-BSA, a trace of which presented on the surface of the GL-BSA. As such, the GL-BSA-HCPT dissolved better than raw HCPT. The drug-release in vitro study indicates that the drug delivery system could release continuously and slowly. Results of the hemolysis test show that the GL-BSA was a safe drug carrier. As both of BSA and GL are biocompatible, feasibility of cytotoxicity assay can be predicted. The amount of GL that coupled to the BSA was $98.26 \mu \mathrm{g} / \mathrm{mg}$.

As GL is mainly taken up into the liver, the liposomes modified by GL on the surface were subject to livertaxis, and the livertaxis of BSA modified by GL has been reported in the literature. This theory was confirmed again by our cell uptake study and cell proliferation assay. The uptake of FITC-labeled samples was observed by confocal microscopy and we found that cells incubated with GL-BSA-HCPT-NPs showed more extensive fluorescence spots and a stronger fluorescence intensity than samples without GL-conjugated NPs.

MTT assay was used to determine the inhibitory rate of samples. The inhibitory rate of GL-BSA-HCPT-NPs was found to develop as the concentration was raised, and it had a higher inhibitory rate at the same concentration and lower IC50 value than other samples.

Thus, this study shows that GL-BSA-HCPT-NPs can target liver tumor cells. In vivo studies on tumor models will be carried out in future. GL-BSA-HCPT-targeting drug delivery will have a broad range of possible applications in liver tumor targeting therapy.

\section{Acknowledgments}

The authors would like to acknowledge financial support from the Fundamental Research Funds for the Central Universities (DL12EA01-02), the Special Fund for Forestry Scientific Research in the Public Interest (201204601), the Forestry Science and Technology Promotion Project ([158]08), and the Agricultural Science and Technology Achievements Transformation Fund Programs of the Ministry of Science and Technology (2011GB23600016).

\section{Disclosure}

The authors report no conflicts of interest in this work.

\section{References}

1. Jaxel C, Kohn KW, Wani MC, Wall ME, Pommier Y. Structure-activity study of the actions of camptothecin derivatives on mammalian topoisomerase I: evidence for a specific receptor site and a relation to antitumor activity. Cancer Res. 1989;49(6):1465-1469.

2. Wang J, Wang R, Li LB. Preparation and properties of hydroxycamptothecin-loaded nanoparticles made of amphiphilic copolymer and normal polymer. $J$ Colloid Interface Sci. 2009;336(2):808-813.

3. Pu X, Sun J, Wang Y, et al. Development of a chemically stable 10-hydroxycamptothecin nanosuspensions. Int J Pharm. 2009;379(1): $167-173$.

4. Zhang C, Ding Y, Yu LL, Ping Q. Polymeric micelle systems of hydroxycamptothecin based on amphiphilic N-alkyl-N-trimethyl chitosan derivatives. Colloids Surf B Biointerfaces. 2007;55(2): 192-199.

5. Widlak P, Li LY, Wang X, Garrard WT. Action of recombinant human apoptotic endonuclease G on naked DNA and chromatin substrates: cooperation with exonuclease and DNase I. J Biol Chem. 2001;276(51):48404-48409.

6. Fu YR, Yi ZJ, Yan YR, Qiu ZY. Hydroxycamptothecin-induced apoptosis in hepatoma SMMC-7721 cells and the role of mitochondrial pathway. Mitochondrion. 2006;6(4):211-217.

7. Sheth P, Sandhu H, Singhal D, Malick W, Shah N, Kislalioglu MS. Nanoparticles in the pharmaceutical industry and the use of supercritical fluid technologies for nanoparticle production. Curr Drug Deliv. 2012;9(3):269-284.

8. O'Leary J, Muggia FM. Camptothecins: a review of their development and schedules of administration. Eur J Cancer. 1998;34(10):1500-1508.

9. Zhao X, Jiang R, Zu Y, et al. Process optimization studies of 10-Hydroxycamptothecin (HCPT)-loaded folate-conjugated chitosan nanoparticles by SAS-ionic crosslink combination using response surface methodology (RSM). Appl Surf Sci. 2012;258(6): 2000-2005.

10. Wei W, Yue ZG, Qu JB, Yue H, Su ZG, Ma GH. Galactosylated nanocrystallites of insoluble anticancer drug for liver-targeting therapy: an in vitro evaluation. Nanomedicine (Lond). 2010;5(4):589-596.

11. Hong M, Zhu S, Jiang Y, Tang G, Pei Y. Efficient tumor targeting of hydroxycamptothecin loaded PEGylated niosomes modified with transferrin. J Control Release. 2009;133(2):96-102.

12. Yang X, Li L, Wang Y, Tan Y. Preparation, pharmacokinetics and tissue distribution of micelles made of reverse thermo-responsive polymers. Int J Pharm. 2009;370(1-2):210-215.

13. Min KH, Park K, Kim YS, et al. Hydrophobically modified glycol chitosan nanoparticles-encapsulated camptothecin enhance the drug stability and tumor targeting in cancer therapy. $J$ Control Release. 2008;127(3):208-218.

14. Bokharaei M, Margaritis A, Xenocostas A, Freeman DJ. Erythropoietin encapsulation in chitosan nanoparticles and kinetics of drug release. Curr Drug Deliv. 2011;8(2):164-171.

15. Mehta AK, Yadav KS, Sawant KK. Nimodipine loaded PLGA nanoparticles: formulation optimization using factorial design, characterization and in vitro evaluation. Curr Drug Deliv. 2007;4(3):185-193.

16. Sun X, Wu F, Lu W, Zhang ZR. Sustained-release hydroxycamptothecin polybutylcyanoacrylate nanoparticles as a liver targeting drug delivery system. Pharmazie. 2004;59(10):791-794.

17. Li Q, Liu C, Zhao X, et al. Preparation, characterization and targeting of micronized 10-hydroxycamptothecin-loaded folate-conjugated human serum albumin nanoparticles to cancer cells. Int J Nanomedicine. 2011;6:397-405.

18. Elzoghby AO, Samy WM, Elgindy NA. Albumin-based nanoparticles as potential controlled release drug delivery systems. J Control Release. 2012;157(2):168-182. 
19. Hu YJ, Liu Y, Sun TQ, Bai AM, Lü JQ, Pi ZB. Binding of antiinflammatory drug cromolyn sodium to bovine serum albumin. Int $J$ Biol Macromol. 2006;39(4-5):280-285.

20. Tantra R, Tompkins J, Quincey P. Characterisation of the deagglomeration effects of bovine serum albumin on nanoparticles in aqueous suspension. Colloids Surf B Biointerfaces. 2010;75(1):275-281.

21. Yang L, Cui F, Cun D, Tao A, Shi K, Lin W. Preparation, characterization and biodistribution of the lactone form of 10-hydroxycamptothecin (HCPT)-loaded bovine serum albumin (BSA) nanoparticles. Int $J$ Pharm. 2007;340(1-2):163-172.

22. Opanasopit P, Yokoyama M, Watanabe M, Kawano K, Maitani Y, Okano T. Block copolymer design for camptothecin incorporation into polymeric micelles for passive tumor targeting. Pharm Res. 2004;21(11):2001-2008.

23. Parkin DM, Bray F, Ferlay J, Pisani P. Estimating the world cancer burden: Globocan 2000. Int J Cancer. 2001;94(2):153-156.

24. Lambert B, Bacher K, Defreyne L, et al. 188Re-HDD/lipiodol therapy for hepatocellular carcinoma: a phase I clinical trial. J Nucl Med. 2005;46(1):60-66.

25. Chakraborty S, Das T, Sarma HD, Venkatesh M, Banerjee S. Preparation and preliminary studies on 177Lu-labeled hydroxyapatite particles for possible use in the therapy of liver cancer. $\mathrm{Nucl} \mathrm{Med} \mathrm{Biol.}$ 2008;35(5):589-597.

26. Law AL, Ng WT, Lee MC, et al. Treatment of primary liver cancer using highly-conformal radiotherapy with $\mathrm{kV}$-image guidance and respiratory control. Radiother Oncol. 2012;102(1):56-61.

27. Nowak AK, Chow PKH, Findlay M. Systemic therapy for advanced hepatocellular carcinoma: a review. Eur J Cancer. 2004; 40(10):1474-1484.

28. Tripathi M, Singh BK, Kakkar P. Glycyrrhizic acid modulates t-BHP induced apoptosis in primary rat hepatocytes. Food Chem Toxicol. 2009;47(2):339-347.

29. van Rossum TG, Vulto AG, Hop WC, Brouwer JT, Niesters HG, Schalm SW. Intravenous glycyrrhizin for the treatment of chronic hepatitis C: a double-blind, randomized, placebo-controlled phase I/II trial. J Gastroenterol Hepatol. 1999;14(11):1093-1099.

30. Ploeger B, Mensinga T, Sips A, Seinen W, Meulenbelt J, DeJongh J. The pharmacokinetics of glycyrrhizic acid evaluated by physiologically based pharmacokinetic modeling. Drug Metab Rev. 2001;33(2): 125-147.

31. Tsuji H, Osaka S, Kiwada H. Targeting of liposomes surface-modified with glycyrrhizin to the liver. I. Preparation and biological disposition. Chem Pharm Bull (Tokyo). 1991;39(4):1004-1008.

32. Osaka S, Tsuji H, Kiwada H. Uptake of liposomes surface-modified with glycyrrhizin by primary cultured rat hepatocytes. Biol Pharm Bull. 1994;17(7):940-943.

33. Lin A, Chen J, Liu Y, et al. Preparation and evaluation of N-caproyl chitosan nanoparticles surface modified with glycyrrhizin for hepatocyte targeting. Drug Dev Ind Pharm. 2009;35(11):1348-1355.

34. Mao S, Hou S, Zhang L, et al. Preparation of valaciclovir loaded bovine serum albumin nanoparticles surface-modified with glycyrrhizin and its characteristics of targeting to liver. Sheng Wu Yi Xue Gong Cheng Xue Za Zhi. 2004;21(4):570-574. Chinese.
35. Institute for Laboratory Animal Research. Guide for the Care and Use of Laboratory Animals. National Institutes of Health Publication No 85-23. Washington DC: National Academies Press; 1996.

36. Zhang L, Sun Y, Chen T, Xu Q. Selective depletion of glycyrrhizin from Si-Ni-San, a traditional Chinese prescription, blocks its effect on contact sensitivity in mice and recovers adhesion and metalloproteinases production of T lymphocytes. Int Immunopharmacol. 2005;5(7-8):1193-1204.

37. Zu Y, Zhang Y, Zhao X, Zhang Q, Liu Y, Jiang R. Optimization of the preparation process of vinblastine sulfate (VBLS)-loaded folateconjugated bovine serum albumin (BSA) nanoparticles for tumortargeted drug delivery using response surface methodology (RSM). Int J Nanomedicine. 2009;4:321-333.

38. Lian H, Sun J, Yu YP, et al. Supramolecular micellar nanoaggregates based on a novel chitosan/vitamin E succinate copolymer for paclitaxel selective delivery. Int J Nanomedicine. 2011;6:3323-3334.

39. Sun JB, Duan JH, Dai SL, et al. In vitro and in vivo antitumor effects of doxorubicin loaded with bacterial magnetosomes (DBMs) on H22 cells: the magnetic bio-nanoparticles as drug carriers. Cancer Lett. 2007;258(1):109-117.

40. Tcholakova S, Denkov ND, Ivanov IB, Campbell B. Coalescence stability of emulsions containing globular milk proteins. Adv Colloid Interface Sci. 2006;123-126:259-293.

41. Affandi MMM, Julianto T, Majeed A. Development and stability evaluation of astaxanthin nanoemulsion. Asian J Pharm Clin Res. 2011;4:142-148.

42. Obolentseva GV, Litvinenko VI, Ammosov AS, Popova TP, Sampiev AM. Pharmacological and therapeutic properties of licorice preparations (A review). Pharm Chem J. 1999;33(8):427-434.

43. Obolentseva GV, Litvinenko VI, Ammosov AS, Popova TP, Sampiev AM. Pharmacological and therapeutic properties of licorice preparations (A review). Pharmaceutical Chemistry Journal. 1999; 33(8):427-434.

44. Zhao X, Zu Y, Zu S, Wang D, Zhang Y, Zu B. Insulin nanoparticles for transdermal delivery: preparation and physicochemical characterization and in vitro evaluation. Drug Dev Ind Pharm. 2010; 36(10):1177-1185

45. Kim JS, Kim MS, Park HJ, Jin SJ, Lee S, Hwang SJ. Physicochemical properties and oral bioavailability of amorphous atorvastatin hemicalcium using spray-drying and SAS process. Int J Pharm. 2008; 359(1-2):211-219.

46. Grinberg O, Gedanken A, Patra CR, Patra S, Mukherjee P, Mukhopadhyay D. Sonochemically prepared BSA microspheres containing Gemcitabine, and their potential application in renal cancer therapeutics. Acta Biomater. 2009;5(8):3031-3037.

47. Peng YR, Li YB, Liu XD, Zhang JF, Duan JA. Apoptosis induced by caudatin in human hepatoma cell line SMMC7721. Chin J Nat Med. 2008;6(3):210-213.

48. Stockert JC, Blázquez-Castro A, Cañete M, Horobin RW, Villanueva Á. MTT assay for cell viability: Intracellular localization of the formazan product is in lipid droplets. Acta Histochem. 2012;114(8):785-796.
International Journal of Nanomedicine

\section{Publish your work in this journal}

The International Journal of Nanomedicine is an international, peerreviewed journal focusing on the application of nanotechnology in diagnostics, therapeutics, and drug delivery systems throughout the biomedical field. This journal is indexed on PubMed Central, MedLine, CAS, SciSearch $\AA$, Current Contents ${ }^{\circledR} /$ Clinical Medicine,
Dovepress

Journal Citation Reports/Science Edition, EMBase, Scopus and the Elsevier Bibliographic databases. The manuscript management system is completely online and includes a very quick and fair peer-review system, which is all easy to use. Visit http://www.dovepress.com/ testimonials.php to read real quotes from published authors. 